\title{
Characterizing the hyperchaotic dynamics of a semiconductor laser subject to optical feedback via permutation entropy
}

\author{
Luciano Zunino, Osvaldo A. Rosso, and Miguel C. Soriano
}

\begin{abstract}
The time evolution of the output of a semiconductor laser subject to delayed optical feedback can exhibit highdimensional chaotic fluctuations. In this contribution, our aim is to quantify the degree of unpredictability of this hyperchaotic time evolution. To that end, we estimate permutation entropy, a novel information-theory-derived quantifier particularly robust in a noisy environment. The permutation entropy is defined as a functional of a symbolic probability distribution, evaluated using the Bandt-Pompe recipe to assign a probability distribution function to the time series generated by the chaotic system. This measure quantifies the diversity of orderings present in the associated time series. In order to evaluate the performance of this novel quantifier, we compare with the results obtained by using a more standard chaos quantifier, namely the KolmogorovSinai entropy. Here, we present numerical results showing that the permutation entropy, evaluated at specific time-scales involved in the chaotic regime of the semiconductor laser subject to optical feedback, give valuable information about the degree of unpredictability of the chaotic laser dynamics. The influence of additive observational noise on the proposed tool is also investigated.
\end{abstract}

Index Terms-Chaos, semiconductor lasers, optical feedback, degree of unpredictability, permutation entropy.

\section{INTRODUCTION}

Semiconductor lasers are very sensitive to external perturbations such as injection of light from another laser [1], feedback from a distant mirror [2], phase conjugate feedback [3], electro optical feedback [4] or frequency filtered external optical feedback [5]. As a consequence of these perturbations, the output of these lasers may show very complex dynamical behavior. In particular, high-dimensional chaos can be developed [6], [7].

Chaotic semiconductor lasers are able to synchronize when coupled, and can be used to transmit encrypted information [8]-[12]. In this context, computational tools for assessing the predictability of the associated temporal traces are of extreme importance because they are directly related to security.

L. Zunino and M. C. Soriano are with the Instituto de Física Interdisciplinar y Sistemas Complejos (IFISC) CSIC-UIB, Campus Universitat de les Illes Balears, E-07122 Palma de Mallorca, Spain (e-mail: luciano@ifisc.uib-csic.es; miguel@ifisc.uib-csic.es).

L. Zunino is also with the Centro de Investigaciones Ópticas (CONICET La Plata - CIC), C.C. 3, 1897 Gonnet, Argentina and Departamento de Ciencias Básicas, Facultad de Ingeniería, Universidad Nacional de La Plata (UNLP), 1900 La Plata, Argentina (e-mail: lucianoz@ciop.unlp.edu.ar).

O. A. Rosso is with the Instituto de Ciências Exatas (Fisica), Universidade Federal de Minas Gerais, Av. Antônio Carlos, 6627 - Campus Pampulha, 31270-901 Belo Horizonte - MG, Brazil and Chaos \& Biology Group, Instituto de Cálculo, Facultad de Ciencias Exactas y Naturales, Universidad de Buenos Aires, Pabellón II, Ciudad Universitaria. 1428 Ciudad de Buenos Aires, Argentina (e-mail: oarosso@fibertel.com.ar).
The security of data encryption using techniques based on chaotic carriers relies, to a large extent, on the unpredictability of the carrier signal and needs to be quantified [13]-[15]. It is the purpose of the present article to quantify the unpredictability of the dynamics generated by semiconductor lasers subject to delayed optical feedback as a function of the control system parameters. This analysis can provide useful information for choosing the optimal chaotic carrier.

Semiconductor lasers subject to optical feedback can be modeled via delay differential equations (DDEs) [16], being an example of infinite-dimensional chaotic systems. Although systems with delay are theoretically infinite dimensional, many infinite-dimensional systems can be considered, in practice, to have a finite dimension and can be fully characterized by the largest Lyapunov exponents [7]. The knowledge of the positive Lyapunov exponents allows computing the Kolmogorov-Sinai (KS) entropy through the Pesin's identity, which measures the average loss of information rate and can be seen as a complexity measure of the chaotic attractor. The KS entropy is a widely used invariant of a dynamical system. However, its estimation is highly demanding, particularly for experimental analysis. Lyapunov exponents are very difficult, if not impossible, to be estimated when analyzing data coming from complex systems. Therefore, alternative ways to calculate the KS entropy, instead of applying Pesin's identity, should be taken into consideration [17], [18]. In this work we investigate the properties of a novel measure based on information theory, called permutation entropy (PE) [19], as an alternative to estimate the unpredictability of chaotic time traces. This symbolic entropy quantifier has the advantage to be a straightforward measure and its estimation does not involve demanding and time-consuming numerical calculations. The basic intrinsic structure of complex systems is obtained in a very fast and flexible way. It was shown that the PE based methodology is up to 100 times faster than a Lyapunov exponent based technique, due to the fact that neighborhood searching is not needed [20]. Moreover, PE approach is invariant with respect to nonlinear monotonous transformations [19]. Thus, nonlinear drifts or scalings artificially introduced by a measurement device do not modify the quantifier estimations. This property is highly desired for the analysis of experimental data.

It is worth mentioning that a related permutationinformation-theory approach was recently introduced to identify delay phenomena and characteristic time scales from time series [21], [22]. It was shown, both numerically and experimentally, that permutation entropy and permutation statistical 
complexity present well defined extrema when the embedding delay of the symbolic reconstruction matches the intrinsic time scales of the systems dynamics. Estimations of both quantifiers are necessary to identify all the relevant time scales. Moreover, it was found that this methodology is particularly robust in a noise environment [21]. In the present work, and by estimating the permutation entropy from numerically generated time series, we provide evidence that this quantifier can be a practical, simple and efficient alternative for the purpose of quantifying the unpredictability degree of hyperchaotic laser dynamics.

The paper is organized as follows. In Section II we briefly describe the Lang-Kobayashi rate equations used for modeling the dynamical behaviors of semiconductor lasers subject to coherent optical feedback. The permutation entropy quantifier is introduced in Section III. In Section IV, numerical results are presented and discussed. A detailed comparison with the results obtained by estimating the Kolmogorov-Sinai entropy is also included in this section. Finally, some concluding remarks are given in Section V.

\section{RATE EQUation Model}

In this paper, we focus on the chaotic dynamics of a semiconductor laser. More specifically, we consider a single mode laser subject to coherent optical feedback. The data used in our analysis originate from the numerical integration of the Lang-Kobayashi rate equation model [16]. These equations have been shown to be successful in modeling the dynamical behaviors of semiconductor lasers subject to weak to moderate coherent optical feedback, with a single reflection in the external cavity. The equations for the complex slowly varying amplitude of the electric field $E(t)$ and the carrier number inside the cavity $N(t)$ are

$$
\begin{aligned}
& \dot{E}(t)=\frac{1+i \alpha}{2}\left[G(t)-\frac{1}{\tau_{p}}\right] E(t)+\gamma E\left(t-\tau_{S}\right) e^{-i \Phi}(1) \\
& \dot{N}(t)=\frac{I}{e}-\frac{N(t)}{\tau_{N}}-G(t)|E(t)|^{2},
\end{aligned}
$$

where $G(t)=g\left(N(t)-N_{0}\right) /\left(1+s|E(t)|^{2}\right)$ is the optical gain, $\alpha$ is the linewidth enhancement factor, $\tau_{p}$ is the photon lifetime, $\tau_{N}$ is the carrier lifetime, $g$ is the differential gain coefficient, $N_{0}$ is the carrier number at transparency, $s$ is the gain compression coefficient, $\tau_{S}$ is the feedback time delay, $\gamma$ is the feedback strength, $\Phi$ is the optical feedback phase and $e$ is the electron charge. $I$ denotes the injected current. Table I details the parameter values that were used in the simulations. With these parameter values, the relaxation oscillation frequency of the solitary laser is $f_{R O}=4.2 \mathrm{GHz}$ at $I=1.5 I_{t h}$.

Time series representing the intensity of the laser were numerically integrated using a second-order Runge-Kutta method with a time step of $\Delta t=0.1 \mathrm{ps}$. In this work ten realizations, each one of length $N=10^{5}$ data points with a sampling period of $\Omega_{s}=1 \mathrm{ps}$, were analyzed in order to obtain statistically significant results. Each realization starts at a different initial condition. Figure 1(a) shows a typical temporal trace of $|E(t)|^{2}$ generated by simulating the Lang-Kobayashi model at
TABLE I

PARAMETER SET IN THE NUMERICAL SIMULATION.

\begin{tabular}{|c|c|c|}
\hline Parameter & Description & Value \\
\hline$\alpha$ & linewidth enhancement factor & 5 \\
$\tau_{p}$ & photon lifetime & $2 \mathrm{ps}$ \\
$\tau_{N}$ & carrier lifetime & $2 \mathrm{~ns}$ \\
$g$ & differential gain coefficient & $1.5 \times 10^{-8} \mathrm{ps}^{-1}$ \\
$N_{o}$ & carrier transparency & $1.5 \times 10^{8}$ \\
$s$ & gain compression coefficient & $5 \times 10^{-7}$ \\
$\tau_{S}$ & feedback time delay & $1 \mathrm{~ns}$ \\
$\gamma$ & feedback strength & $7.5 \mathrm{~ns}^{-1}-40 \mathrm{~ns}^{-1}$ \\
$\Phi$ & optical feedback phase & 0 \\
$I_{t h}$ & threshold current & $14.7 \mathrm{~mA}$ \\
$I$ & injected current & $1.1 I_{t h}-2.5 I_{t h}$ \\
\hline
\end{tabular}

the coherence collapse regime $\left(I=1.5 I_{t h}, \gamma=20 \mathrm{~ns}^{-1}\right)$ and Fig. 1(b) depicts its corresponding autocorrelation function.

\section{Permutation entropy}

Entropy quantifiers are able to capture the degree of uncertainty and disorder present in a time series. Particularly, Shannon entropy is very often used as the first natural entropy measure. Given any arbitrary probability distribution $P=\left\{p_{i}: i=1, \ldots, M\right\}$, the Shannon's logarithmic information measure, $S[P]=-\sum_{i=1}^{M} p_{i} \ln p_{i}$, is related to the uncertainty associated with the physical process described by $P$. If $S[P]=0$ we are in position to predict with complete certainty which of the possible outcomes $i$ whose probabilities are given by $p_{i}$ will actually take place. In this case, our knowledge of the underlying process described by the probability distribution is maximal. On the contrary, our knowledge is minimal for a uniform distribution and $S[P]$ is maximized.

In order to evaluate the above-mentioned quantifier, $S[P]$, an associated probability distribution should be constructed in advance. The adequate choice of the probability distribution associated to a time series is a crucial step for obtaining a successful characterization of the system. Bandt and Pompe [19] introduced a simple and robust method to evaluate the probability distribution taking into account the time causality of the system dynamics. They suggested that the symbol sequence should arise naturally from the time series, without any model assumptions. Thus, they took partitions by comparing the order of neighboring values rather than partitioning the amplitude into different levels. That is, given a time series $\left\{x_{t}, t=1, \ldots, N\right\}$, an embedding dimension $D>1(D \in \mathbb{N})$, and an embedding delay $\tau_{e}\left(\tau_{e} \in \mathbb{N}\right)$, the ordinal pattern of order $D$ generated by

$$
s \mapsto\left(x_{s-(D-1) \tau_{e}}, x_{s-(D-2) \tau_{e}}, \ldots, x_{s-\tau_{e}}, x_{s}\right)
$$

has to be considered. To each time $s$ we assign a $D$ dimensional vector that results from the evaluation of the time series at times $s-(D-1) \tau_{e}, \ldots, s-\tau_{e}, s$. Clearly, the higher the value of $D$, the more information about the past is incorporated into the ensuing vectors. By the ordinal pattern 

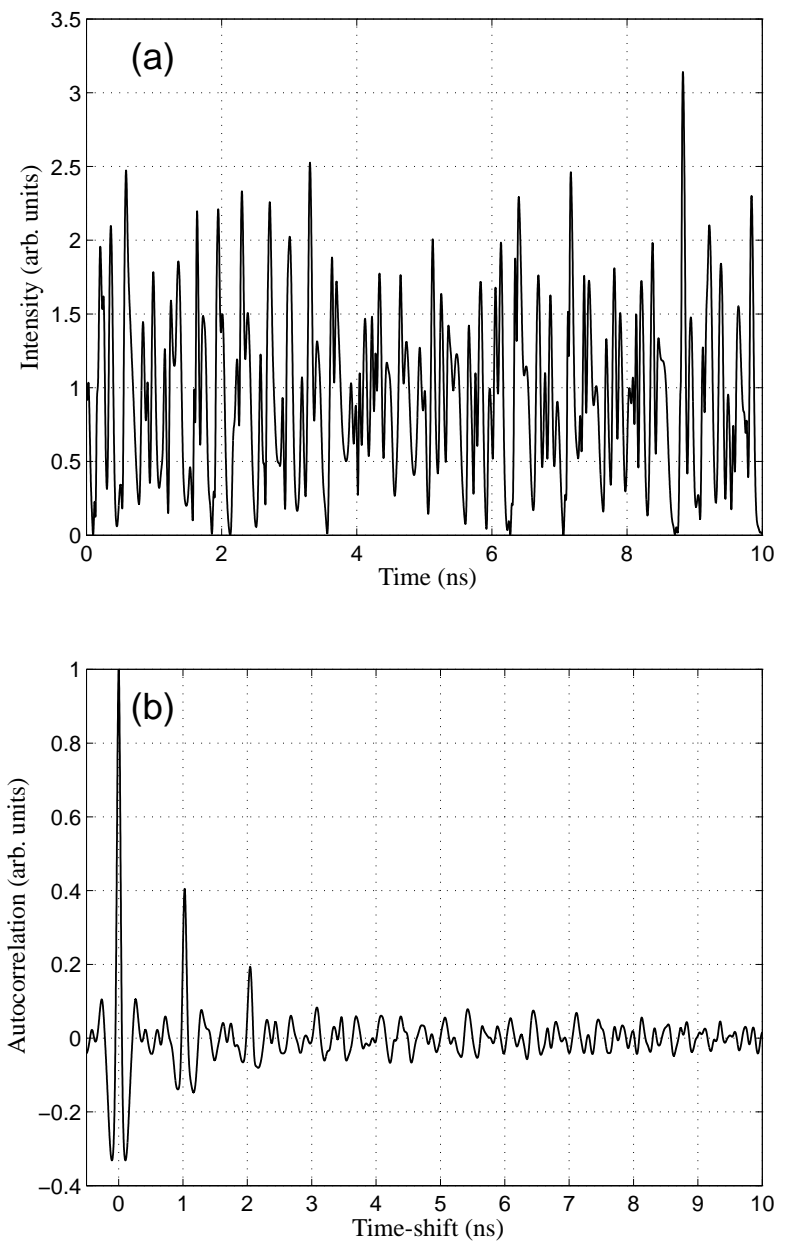

Fig. 1. (a) Temporal evolution of the semiconductor laser intensity $\left(|E(t)|^{2} / 10^{5}\right)$ simulated by using the Lang-Kobayashi model at the coherence collapse regime $\left(I=1.5 I_{t h}, \gamma=20 \mathrm{~ns}^{-1}\right)$. (b) Autocorrelation function of the semiconductor laser intensity $|E(t)|^{2}$.

of order $D$ related to the time $s$ we mean the permutation $\pi=\left(r_{0}, r_{1}, \ldots, r_{D-1}\right)$ of $(0,1, \ldots, D-1)$ defined by

$$
x_{s-r_{0} \tau_{e}} \geq x_{s-r_{1} \tau_{e}} \geq \cdots \geq x_{s-r_{D-2} \tau_{e}} \geq x_{s-r_{D-1} \tau_{e}} .
$$

In this way the vector defined by Eq. (3) is converted into a unique symbol $\pi$. The procedure can be better illustrated with a simple example; let us assume that we start with the time series depicted in Fig. 2. It corresponds to a small segment of the semiconductor laser intensity depicted in Fig. 1 but with sampling period of $\Omega_{s}=30 \mathrm{ps}$. We set the embedding dimension $D=4, s=20$ and the embedding delay $\tau_{e}=3$. In this case the state space is divided into 4 ! partitions and 24 mutually exclusive permutation symbols are considered. According to Eq. (3), the first 4-dimensional vector corresponding with $\left(x_{s-3 \tau_{e}}, x_{s-2 \tau_{e}}, x_{s-\tau_{e}}, x_{s}\right)$ is $(0.834,1.537,1.715,1.125)$, see the dashed line in Fig. 2. Following Eq. (4), we find that $x_{s-\tau_{e}} \geq x_{s-2 \tau_{e}} \geq x_{s} \geq x_{s-3 \tau_{e}}$. Then, the ordinal pattern which allows us to fulfill Eq. (4) will be $(1,2,0,3)$. The next 4dimensional vector, at $s=21$, is $(0.864,0.640,1.128,1.287)$, and $(0,1,3,2)$ will be its associated permutation, and so on. In order to get a unique result we consider that $r_{i}<r_{i-1}$

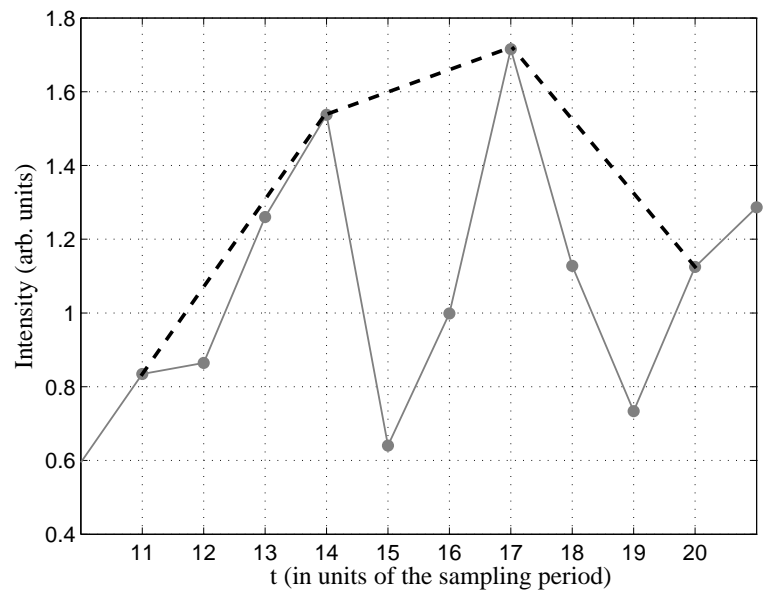

Fig. 2. Procedure to identify ordinal patterns from a small segment of the semiconductor laser intensity time series $\left(|E(t)|^{2} / 10^{5}\right)$ depicted in Fig. 1 but with sampling period of $\Omega_{s}=30 \mathrm{ps}$. In this particular example embedding dimension $D=4$, embedding delay $\tau_{e}=3$ and time $s=20$ are considered

if $x_{s-r_{i} \tau_{e}}=x_{s-r_{i-1} \tau_{e}}$. This is justified if the values of $x_{t}$ have a continuous distribution so that equal values are very unusual. Otherwise, it is possible to break these equalities by adding small random perturbations [19].

For all the $D$ ! possible orderings (permutations) $\pi_{i}$ when the embedding dimension is $D$, their associated relative frequencies can be naturally computed by the number of times this particular order sequence is found in the time series divided by the total number of sequences. Thus, an ordinal pattern probability distribution $P=\left\{p\left(\pi_{i}\right), i=1, \ldots, D !\right\}$ is obtained from the time series. It is clear that with this ordinal time series analysis some details of the original amplitude information are lost. However, a meaningful reduction of the complex systems to their basic intrinsic structure is provided. This way of symbolizing time series, based on a comparison of consecutive points, allows a more accurate empirical reconstruction of the underlying phase space of chaotic time series affected by weak (observational and dynamical) noise [19]. Furthermore, the ordinal pattern probability distribution is invariant with respect to nonlinear monotonous transformations. Thus, nonlinear drifts or scalings artificially introduced by a measurement device do not modify the quantifiers' estimations, a relevant property for the analysis of experimental data. These are the main advantages with respect to more conventional methods based on range partitioning. The probability distribution $P$ is obtained once we fix the embedding dimension $D$ and the embedding delay $\tau_{e}$. The former parameter plays an important role for the evaluation of the appropriate probability distribution, since $D$ determines the number of accessible states, given by $D$ !. Moreover, it was established that the length $N$ of the time series must satisfy the condition $N \gg D$ ! in order to achieve a proper differentiation between stochastic and deterministic dynamics [23]. With respect to the selection of the other parameter, Bandt and Pompe specifically considered an embedding delay $\tau_{e}=1$ in their cornerstone paper [19]. Nevertheless, it is clear that other values of $\tau_{e}$ could provide 
additional information.

The normalized permutation entropy is just defined as the normalized Shannon entropy associated to the Bandt and Pompe probability distribution $P=\left\{p\left(\pi_{i}\right), i=1, \ldots, D !\right\}$ :

$$
\mathcal{H}_{S}[P]=S[P] / S_{\max }=\left[-\sum_{i=1}^{D !} p\left(\pi_{i}\right) \ln \left(p\left(\pi_{i}\right)\right)\right] / S_{\max },
$$

where $S_{\max }=\ln D$ !, $\left(0 \leq \mathcal{H}_{S} \leq 1\right)$, and $S$ stands for Shannon entropy. The lower bound is reached for regular dynamics (e.g., periodic) and the upper bound for completely random stochastic dynamics (i.e., white noise). This symbolic entropy measure, that quantifies the diversity of orderings, was shown to be particularly useful for very different purposes like detecting dynamical changes in complex time series [20], predicting epileptic seizures [24], characterizing stochastic processes [25], tracking the anesthetic drug effect in electroencephalographic data [26], testing for independence [27], detecting noise-induced temporal correlations in stochastic resonance phenomena [28], measuring the stock market inefficiency [29], and quantifying the randomness of chaotic pseudo-random number generators [30]. In the next section, we show that this information-theory-derived quantifier can also be useful for detecting and quantifying the presence of correlations and regular structures in the time traces of high-dimensional chaotic systems. The smaller the value of $\mathcal{H}_{S}$, the more regular the time series is. The identification of regularities between subsequent points of a time series by using the symbolic Bandt and Pompe approach was recently shown specially useful for distinguishing chaotic sequences from random ones [31]-[33].

\section{NUMERICAL RESUltS}

\section{A. Permutation entropy approach}

In this section, we investigate the unpredictability of the hyperchaotic laser dynamics by estimating the permutation entropy, $\mathcal{H}_{S}$, to quantify the presence of patterns and regular structures in the chaotic time series. The main parameters involved in the numerical computation of $\mathcal{H}_{S}$ are the embedding dimension, $D$, and the embedding delay, $\tau_{e}$. In the present study, we consider different embedding dimensions $4 \leq D \leq 7$, and we vary $\tau_{e}$ between 1 and 2000. We have found that the results obtained are strongly dependent on the embedding delay. Therefore, we advise a proper selection of this parameter for obtaining meaningful results. The embedding delay $\tau_{e}$ is directly related to the sampling frequency of the system under analysis [34]. By increasing this parameter, the original time series is subsampled in a very efficient way. It has been recently shown that characteristic time scales present in the system dynamics are detected through the presence of clear extrema of the permutation entropy and permutation complexity when they are calculated as a function of the embedding delay [21], [22]. Furthermore, periodicities present in the system can be identified by analyzing the behavior of the permutation entropy as a function of $\tau_{e}$ [35].

We first present the results for $\mathcal{H}_{S}$ for different embedding dimensions $(4 \leq D \leq 7)$ with a $\tau_{e}$ value that typically allows for a proper reconstruction of the chaotic attractor. This optimal reconstruction lag, denoted hereafter as $\tau_{e}^{\text {rec }}$, is estimated from the autocorrelation function at the next point where it drops to $1-e^{-1} \approx 0.63$ of its initial value [36], [37]. Estimated values for $\tau_{e}^{\text {rec }}$ as a function of the injected current and feedback strength are detailed in Table II. The values obtained for this optimal reconstruction lag are approximately a tenth of the solitary laser relaxation oscillation period. Since the sampling time of the laser intensity time series is $\Omega_{s}=1 \mathrm{ps,}$ $\tau_{e}=\tau_{e}^{\text {rec }}$ captures the semiconductor laser dynamical behavior at the fastest time-scale of Eqs. (1)-(2), which is given by the photon lifetime (picoseconds). Figures 3(a) and (b) show $\mathcal{H}_{S}$, evaluated at $\tau_{e}=\tau_{e}^{\text {rec }}$, as a function of the injected current (for a fixed feedback strength $\gamma=20 \mathrm{~ns}^{-1}$ ) and the feedback strength (for a fixed injected current $I=1.5 I_{t h}$ ), respectively. Mean and standard deviation of the permutation entropy estimations obtained for the ten different realizations for each control parameter value are plotted. On the one hand, we find that $\mathcal{H}_{S}$ decreases with an increasing injected current. On the other hand, we find that $\mathcal{H}_{S}$ increases with an increasing feedback strength, saturating at large values of $\gamma$.

TABLE II

$\tau_{e}^{\text {rec }}$ MEASURED IN UNITS OF THE SAMPLING PERIOD $\left(\Omega_{s}=1 \mathrm{ps}\right)$ FOR DIFFERENT INJECTED CURRENTS $\left(\gamma=20 \mathrm{~ns}^{-1}\right)$ AND DIFFERENT FEEDBACK STRENGTHS $\left(I=1.5 I_{t h}\right)$. MEAN VALUES ASSOCIATED TO THE TEN DIFFERENT REALIZATIONS ARE DETAILED.

\begin{tabular}{|c|c|c|c|}
\hline$I / I_{t h}$ & $\tau_{e}^{\text {rec }}(\mathrm{ps})$ & $\gamma\left(\mathrm{ns}^{-1}\right)$ & $\tau_{e}^{\mathrm{rec}}(\mathrm{ps})$ \\
\hline 1.1 & 37 & 7.5 & 30 \\
\hline 1.2 & 31 & 10 & 28 \\
\hline 1.3 & 28 & 12.5 & 26 \\
\hline 1.4 & 26 & 15 & 26 \\
\hline 1.5 & 24 & 17.5 & 25 \\
\hline 1.6 & 23 & 20 & 24 \\
\hline 1.7 & 21 & 22.5 & 23 \\
\hline 1.8 & 20 & 25 & 23 \\
\hline 1.9 & 19 & 27.5 & 22 \\
\hline 2.0 & 18 & 30 & 21 \\
\hline 2.1 & 18 & 32.5 & 20 \\
\hline 2.2 & 17 & 35 & 19 \\
\hline 2.3 & 17 & 37.5 & 18 \\
\hline 2.4 & 16 & 40 & 18 \\
\hline 2.5 & 16 & & \\
\hline
\end{tabular}

We also present the results for $\mathcal{H}_{S}$ when $\tau_{e}=1000$, which matches a characteristic time-scale of the system, namely the feedback time delay $\left(\tau_{e}=\tau_{S} / \Omega_{s}=1000\right)$. Figure 4(a) shows $\mathcal{H}_{S}$ as a function of the injected current for a constant feedback strength $\left(\gamma=20 \mathrm{~ns}^{-1}\right)$. A clear maximum can be seen at $I \approx 1.4 I_{t h}$. In Fig. 4(b), $\mathcal{H}_{S}$ as a function of the feedback strength for a constant injected current $(I=$ $\left.1.5 I_{t h}\right)$ is depicted. We find that $\mathcal{H}_{S}$ has a maximum at $\gamma \approx 20 \mathrm{~ns}^{-1}$ when $\tau_{e}=1000$. We have found a similar behavior when the embedding delay $\tau_{e}$ matches harmonics and some subharmonics of the system's feedback time delay $\tau_{S}$. More precisely, when $\tau_{e}=k \tau_{S} / \Omega_{s}$ with $k \in \mathbb{N}$ and when 

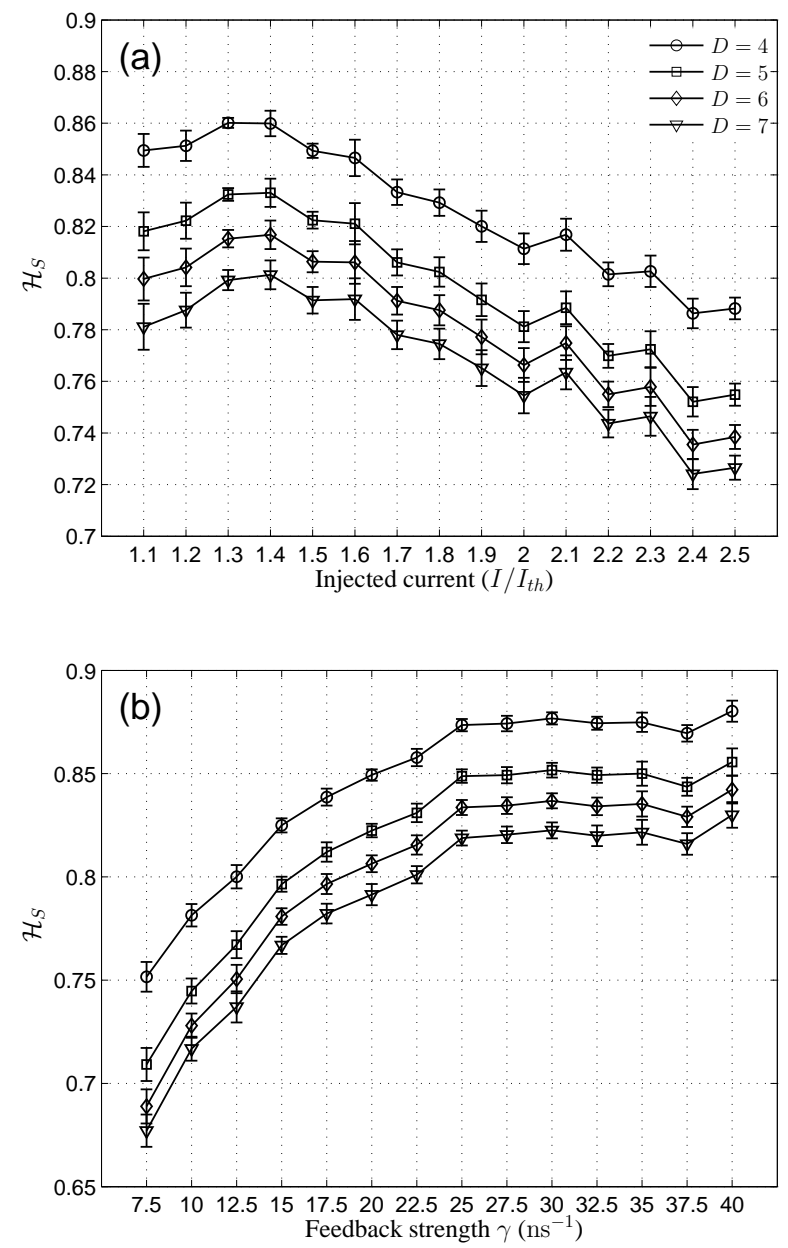

Fig. 3. Permutation entropy for the semiconductor laser subject to optical feedback as a function of (a) injected current $\left(\gamma=20 \mathrm{~ns}^{-1}\right)$ and (b) feedback strength $\left(I=1.5 I_{t h}\right)$. Embedding delay $\tau_{e}=\tau_{e}^{\text {rec }}$ and sampling time $\Omega_{s}=$ 1 ps. Mean and standard deviation of the permutation entropy estimations obtained for ten different realizations are plotted.

$\tau_{e}=\tau_{S} /\left(2 \Omega_{s}\right), \tau_{S} /\left(3 \Omega_{s}\right), \ldots, \tau_{S} /\left((D-1) \Omega_{s}\right)$.

Taking into account the high variability observed in the permutation entropy estimations as a function of the embedding delay $\tau_{e}$, it is essential to compare with the results obtained by using a more established tool. In the next subsection we detail the results obtained for the Kolmogorov-Sinai (KS) entropy, a well-known and widely used invariant of a dynamical system.

\section{B. Kolmogorov-Sinai Entropy}

The Kolmogorov-Sinai (KS) entropy measures the average loss of information rate. Its range of values goes from zero for regular dynamics, it is positive for chaotic systems and infinite for a perfectly stochastic process. The larger the entropy, the larger the unpredictability of the system, which is a highly desired property to ensure security in a chaos encryption scheme. The computation of the KS entropy is performed from the positive Lyapunov exponents as follows (see Ref. [38], pp.
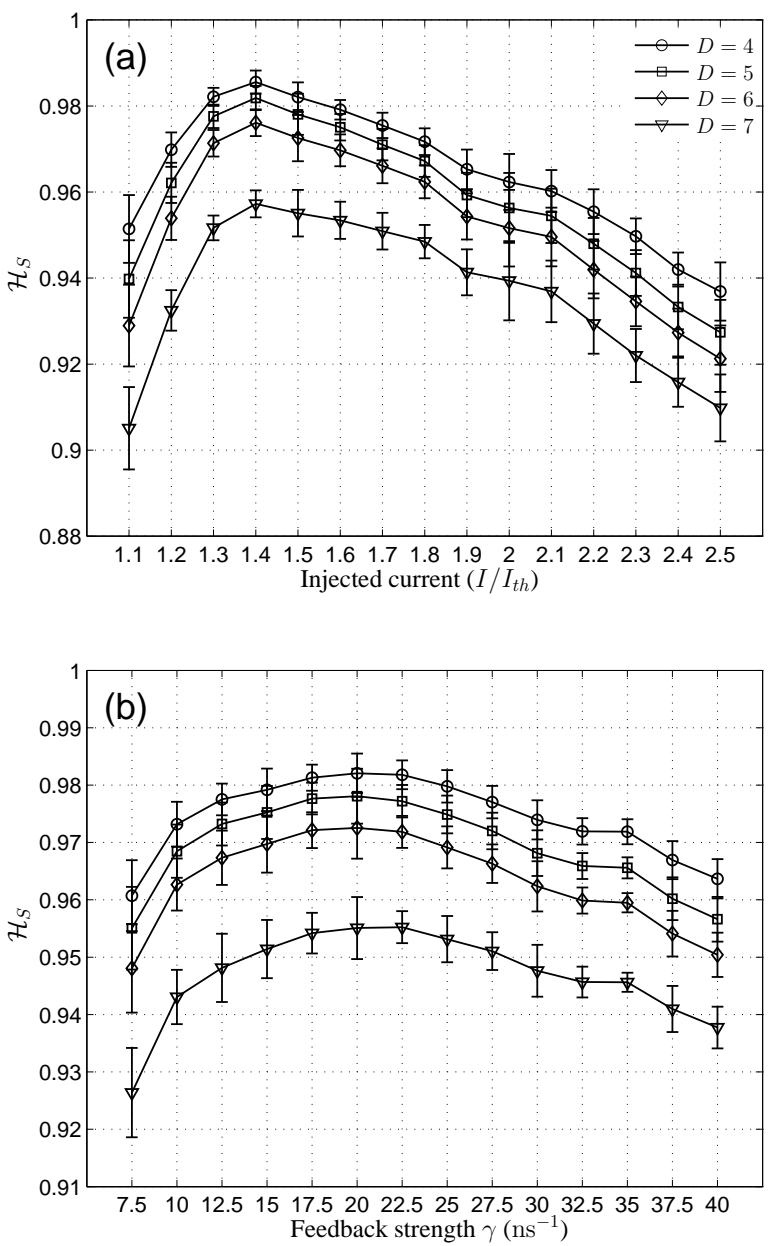

Fig. 4. Permutation entropy for the semiconductor laser subject to optical feedback as a function of (a) injected current $\left(\gamma=20 \mathrm{~ns}^{-1}\right)$ and (b) feedback strength $\left(I=1.5 I_{t h}\right)$. Embedding delay $\tau_{e}=\tau_{S} / \Omega_{s}=1000$ and sampling time $\Omega_{s}=1$ ps. Mean and standard deviation of the permutation entropy estimations obtained for ten different realizations are plotted.

229-231)

$$
h_{K S}=\sum_{i \mid \lambda_{i}>0} \lambda_{i}
$$

where $\lambda_{i}$ are the Lyapunov exponents.

To be precise, the sum of the positive Lyapunov exponents is an upper bound to the Kolmogorov-Sinai entropy but Eq. (6) seems to hold in very general situations and it is usually the only way to obtain a good estimation of $h_{K S}$ [7]. The Lyapunov exponents have been computed following Farmer's approach [39]. We have integrated the corresponding delay differential equations with an Euler method. Therefore, the original delay differential equations are converted into a map. We then compute the Lyapunov exponents of this map. Only a finite portion of the set of $\lambda_{i}$ can be determined by such a numerical analysis. The number of Lyapunov exponents that can be calculated is given by $\tau_{S} / h$, where $h$ is the discretization time step and $\tau_{S}$ is the feedback time delay. The results for the largest Lyapunov exponents are independent of the time step provided that $h$ is not too large. The KS 

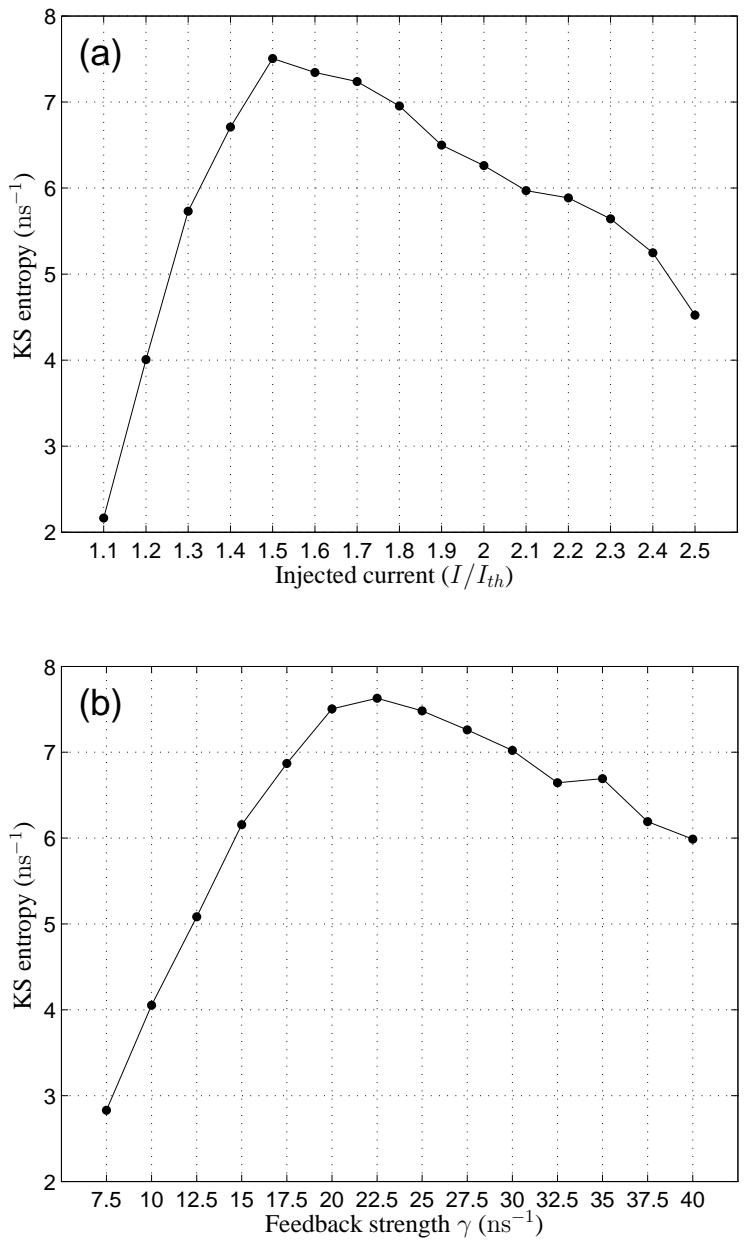

Fig. 5. KS entropy for the semiconductor laser subject to optical feedback as a function of (a) injected current $\left(\gamma=20 \mathrm{~ns}^{-1}\right)$ and (b) feedback strength $\left(I=1.5 I_{t h}\right)$.

entropy is characterized by the positive $\lambda_{i}$ obtained with this procedure [7], [39].

In Fig. 5(a), we present the KS entropy as a function of the injected current for a constant feedback strength $\gamma=20 \mathrm{~ns}^{-1}$. It can be seen the KS entropy displays a maximum around $I=1.5 I_{t h}$. We also show the KS entropy as a function of the feedback strength in Fig. 5(b) for a constant injected current $I=1.5 I_{t h}$. The KS entropy increases with an increasing feedback strength up to $\gamma \approx 22.5 \mathrm{~ns}^{-1}$, where it reaches a maximum. The results presented in this section for the KS entropy quantitatively agree with the results presented by Vicente et al. [7], where the hyperchaoticity of the laser dynamics was described by means of the KS entropy and the Kaplan-Yorke dimension.

It is clear that the results obtained for $\mathcal{H}_{S}$ and for the KS entropy differ when the former quantifier is estimated by considering $\tau_{e}=\tau_{e}^{\text {rec }}$, as can be concluded from the comparison of Figs. 3 and 5. However, it has been shown that the KS entropy and the permutation entropy give similar results when the latter is estimated by using an embedding delay $\tau_{e}$ that coincides with the system's feedback time delay
$\tau_{S}$. The same similarity is found when the embedding delay $\tau_{e}$ matches harmonics and some subharmonics of $\tau_{S}$. These results allow us to conclude that the dynamics at the feedback time delay strongly influences the overall dynamical behavior. This finding agrees with the fact that the dynamics of certain chaotic delayed systems can be identified and modeled once their feedback time delay is known [40]-[42].

We have also studied the effect that the feedback time delay $\tau_{S}$ has on the permutation entropy estimations. For that purpose numerical simulations with different feedback time delays were generated $\left(\tau_{S}=100 \mathrm{ps}, 200 \mathrm{ps}, \ldots, 1000 \mathrm{ps}\right)$. Ten different realizations of $N=10^{5}$ data points with a sampling period of $\Omega_{s}=1 \mathrm{ps}$ were considered for each value of the feedback time delay. The injected current and feedback strength were fixed equal to $1.5 I_{t h}$ and $20 \mathrm{~ns}^{-1}$, respectively, for this analysis. Taking into account the previous results we have estimated $\mathcal{H}_{S}$ for $\tau_{e}=\tau_{S} / \Omega_{s}$. The results obtained for $\mathcal{H}_{S}$ are compared with those for the KS entropy in Fig. 6. The trends observed in the curves for the permutation entropy, Fig. 6(a), are reproduced in the curve for the KS entropy, Fig. 6(b). Both the permutation and the KS entropy quantifiers show a constant value for large feedback times.

Finally, we have tested the effect an additive observational noise has on the proposed approach. Taking into account the low sensitivity to noise of the symbolic Bandt and Pompe approach [19], robustness to noise is expected by using the PE quantification. In order to perform this analysis, Gaussian white noises of different noise to signal ratio (NSR) were added to the original semiconductor laser intensity time series. The NSR is defined as the standard deviation of the noise over the standard deviation of the original signal. Figure 7 shows the influence this observational noise has on PE estimations as a function of the injected current, for a fixed feedback strength $\gamma=20 \mathrm{~ns}^{-1}$ and feedback time delay $\tau_{S}=1 \mathrm{~ns}$. Ten different realizations for each $I / I_{t h}$ value were analyzed. Embedding dimension $D=6$ and $\tau_{e}=\tau_{S} / \Omega_{s}=1000$ were chosen for estimating $\mathcal{H}_{S}$. It can be concluded that PE is able to identify the optimal chaotic carrier, obtained at $I \approx 1.4 I_{t h}$, even when the standard deviation of the noise and the original signal are equal (NSR =1). We have found a similar insensitivity to noise in the analysis of $\mathcal{H}_{S}$ as a function of the feedback strength for a constant injected current $\left(I=1.5 I_{t h}\right)$. These results confirm the robustness to noise of the permutation entropy approach.

\section{Conclusions}

We have numerically analyzed the degree of unpredictability of the hyperchaotic time traces generated by the output of a semiconductor laser subject to delayed optical feedback by estimating a novel information-theory-derived quantifier, namely the permutation entropy. The behavior of this quantifier as a function of the embedding delay $\tau_{e}$ was analyzed. A detailed comparison with the results obtained by employing the Kolmogorov-Sinai entropy allows us to conclude that the overall laser dynamics is strongly dominated by the delayed feedback term in Eq. (1). More precisely, we found that the permutation entropy should be estimated with an embedding delay $\tau_{e}$ around the feedback time delay $\tau_{S}$ in order to recover 

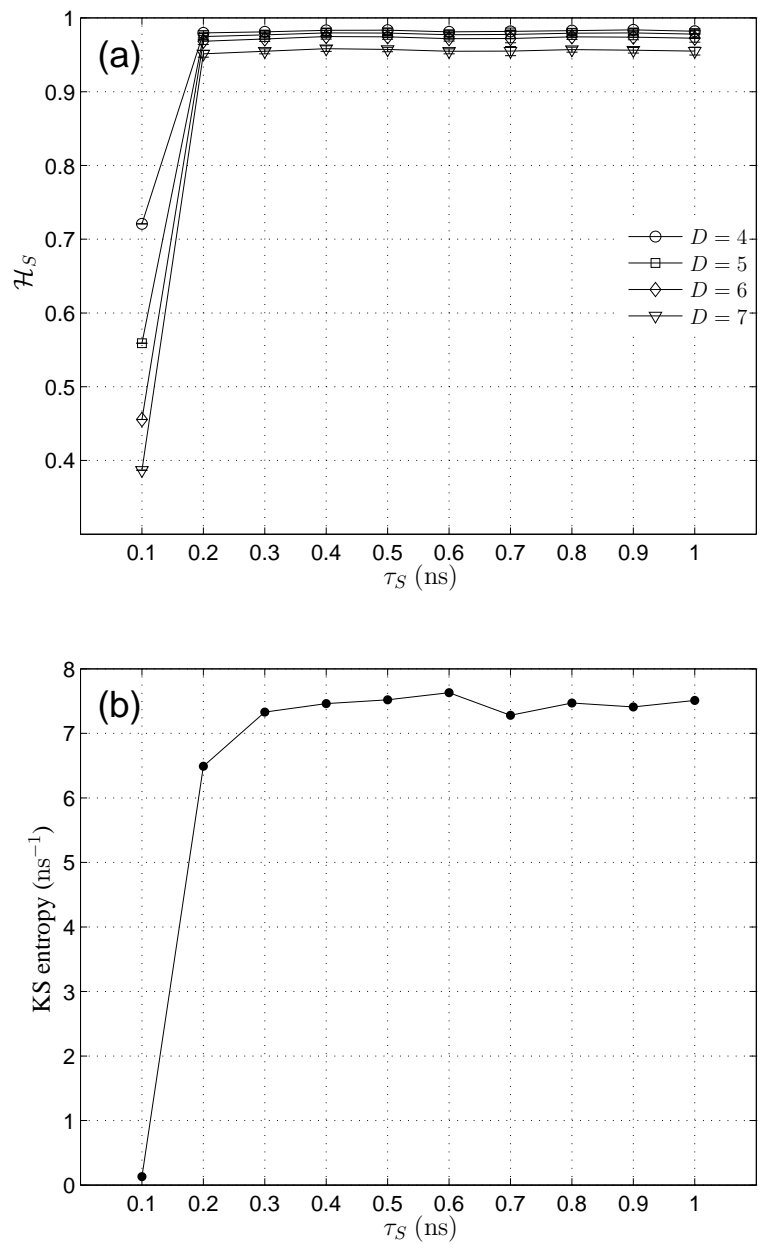

Fig. 6. (a) Permutation entropy and (b) KS entropy for the semiconductor laser subject to optical feedback as a function of the feedback time delay $\tau_{S}\left(\gamma=20 \mathrm{~ns}^{-1}\right.$ and $\left.I=1.5 I_{t h}\right)$. Embedding delay $\tau_{e}=\tau_{S} / \Omega_{s}$ and sampling time $\Omega_{s}=1 \mathrm{ps}$ were considered for the permutation entropy estimations. Mean and standard deviation of the values obtained for ten different realizations are plotted in the case of $\mathcal{H}_{S}$.

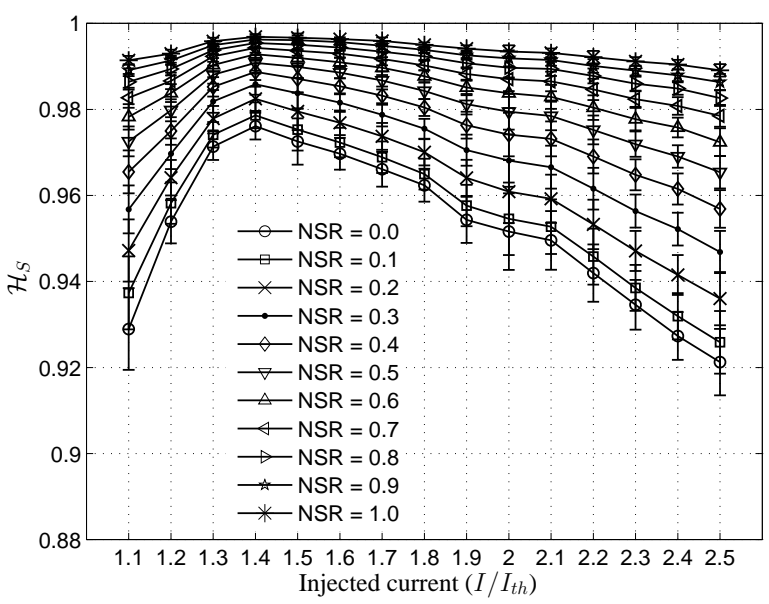

Fig. 7. Permutation entropy for the semiconductor laser subject to optical feedback as a function of the injected current $\left(\gamma=20 \mathrm{~ns}^{-1}\right.$ and $\left.\tau_{S}=1 \mathrm{~ns}\right)$ for different noise to signal ratio (NSR). Embedding dimension $D=6$ and $\tau_{e}=\tau_{S} / \Omega_{s}=1000$ were used for the PE estimations. Mean and standard deviation of the values obtained for ten different realizations are plotted. the results obtained with the KS entropy. We have also checked that this symbolic entropy quantifier is especially robust to additive observational noise. Consequently, its estimation can be very useful for analyzing experimental data.

An exact determination of the Kolmogorov-Sinai entropy is only possible in a few special cases and its estimation from the output time series of a system is very difficult and not free from arbitrariness [43]. In this context, the permutation entropy is an interesting alternative because it can be easily estimated for any type of time series (regular, chaotic, noisy) [19], [44]. Moreover, it was theoretically shown that both quantifiers, i.e. Kolmogorov-Sinai and permutation entropies, coincide for a simple class of dynamical systems [45]. Therefore, we suggest that the permutation entropy can be of more general and practical applicability for arbitrary real-world data. In particular, we have shown that it can be very useful for quantifying the degree of unpredictability of hyperchaotic time traces generated by semiconductor lasers subject to delayed optical feedback. A generalization of this finding to other dynamical systems with delay still remains an open question and needs further study.

\section{ACKNOWLEDGMENT}

We wish to thank Prof. Claudio R. Mirasso and Prof. Ingo Fischer for very useful discussions and comments on the current manuscript. L.Z. and O.A.R. were supported by Consejo Nacional de Investigaciones Científicas y Técnicas (CONICET), Argentina. O.A.R. is PVE fellowship, CAPES, Brazil. Part of this work was funded by MEC (Spain), MICINN (Spain) and FEDER under Projects TEC2009-14101 (DeCoDicA) and FIS2007-60327 (FISICOS), and by the EC Project PHOCUS Grant 240763.

\section{REFERENCES}

[1] S. Wieczorek, B. Krauskopf, and D. Lenstra, "A unifying view of bifurcations in a semiconductor laser subject to optical injection," Opt. Commun., vol. 172, pp. 279-295, 1999.

[2] J. Mørk, B. Tromborg, and J. Mark, "Chaos in semiconductor lasers with optical feedback: Theory and experiment," IEEE J. Quantum Electron., vol. 28, pp. 93-108, 1992.

[3] D. H. DeTienne, G. R. Gray, G. P. Agrawal, and D. Lenstra, "Semiconductor laser dynamics for feedback from a finite-penetration-depth phase-conjugate mirror," IEEE J. Quantum Electron., vol. 33, pp. 838844, 1997.

[4] J.-P. Goedgebuer, L. Larger, and H. Porte, "Optical cryptosystem based on synchronization of hyperchaos generated by a delayed feedback tunable laser diode," Phys. Rev. Lett., vol. 80, p. 2249, 1998.

[5] M. Yousefi and D. Lenstra, "Dynamical behavior of a semiconductor laser with filtered external optical feedback," IEEE J. Quantum Electron., vol. 35, pp. 970-976, 1999.

[6] I. Fischer, O. Hess, W. Elsässer, and E. Göbel, "High-dimensional chaotic dynamics of an external cavity semiconductor laser," Phys. Rev. Lett., vol. 73, pp. 2188-2191, 1994.

[7] R. Vicente, J. Daudén, P. Colet, and R. Toral, "Analysis and characterization of the hyperchaos generated by a semiconductor laser subject to a delayed feedback loop," IEEE J. Quantum Electron., vol. 41, pp. 541-548, 2005

[8] C. R. Mirasso, P. Colet, and P. Garcia-Fernandez, "Synchronization of chaotic semiconductor lasers: application to encoded communications," IEEE Photon. Technol. Lett., vol. 8, pp. 299-301, 1996.

[9] V. Annovazzi-Lodi, S. Donati, and A. Sciré, "Synchronization of chaotic injected-laser systems and its application to optical cryptography," IEEE J. Quantum Electron., vol. 32, pp. 953-959, 1996.

[10] G. D. VanWiggeren and R. Roy, "Communication with chaotic lasers," Science, vol. 279, pp. 1198-1200, 1998. 
[11] J. Ohtsubo, "Chaos synchronization and chaotic signal masking in semiconductor lasers with optical feedback," IEEE J. Quantum Electron., vol. 38, pp. 1141-1154, 2002.

[12] A. Argyris, D. Syvridis, L. Larger, V. Annovazzi-Lodi, P. Colet, I. Fischer, J. Garca-Ojalvo, C. R. Mirasso, L. Pesquera, and K. A. Shore, "Chaos-based communications at high bit rates using commercial fibreoptic links," Nature, vol. 438, pp. 343-346, 2005.

[13] V. S. Udaltsov, J.-P. Goedgebuer, L. Larger, J.-B. Cuenot, P. Levy, and W. T. Rhodes, "Cracking chaos-based encryption systems ruled by nonlinear time delay differential equations," Phys. Lett. A, vol. 308, pp. 54-60, 2003.

[14] M. C. Soriano, P. Colet, and C. R. Mirasso, "Security implications of open- and closed-loop receivers in all-optical chaos-based communications," IEEE Photon. Technol. Lett., vol. 21, pp. 426-428, 2009.

[15] A. Jacobo, M. C. Soriano, C. R. Mirasso, and P. Colet, "Chaos-based optical communications: encryption versus nonlinear filtering," IEEE $J$. Quantum Electron., vol. 46, pp. 499-505, 2010.

[16] R. Lang and K. Kobayashi, "External optical feedback effects on semiconductor injection laser properties," IEEE J. Quantum Electron., vol. 16 , pp. $347-355,1980$

[17] M. S. Baptista, E. J. Ngamga, P. R. F. Pinto, M. Brito, and J. Kurths, "Kolmogorov-Sinai entropy from recurrence times," Physics Letters A, vol. 374, pp. 1135-1140, 2010.

[18] A. W. Jayawardena, P. Xu, and W. K. Li, "Modified correlation entropy estimation for a noisy chaotic time series," Chaos, vol. 20, p. 023104 2010.

[19] C. Bandt and B. Pompe, "Permutation entropy: a natural complexity measure for time series," Phys. Rev. Lett., vol. 88, p. 174102, 2002.

[20] Y. Cao, W. Tung, J. B. Gao, V. A. Protopopescu, and L. M. Hively, "Detecting dynamical changes in time series using the permutation entropy," Phys. Rev. E, vol. 70, p. 046217, 2004.

[21] L. Zunino, M. C. Soriano, I. Fischer, O. A. Rosso, and C. R. Mirasso, "Permutation-information-theory approach to unveil delay dynamics from time-series analysis," Phys. Rev. E, vol. 82, p. 046212, 2010.

[22] M. C. Soriano, L. Zunino, O. A. Rosso, I. Fischer, and C. R. Mirasso, "Time scales of a chaotic semiconductor laser with optical feedback under the lens of a permutation information analysis," IEEE J. Quantum Electron., vol. 47, pp. 252-261, 2011.

[23] M. Staniek and K. Lehnertz, "Parameter selection for permutation entropy measurements," Int. J. of Bifurcation and Chaos, vol. 17, pp. 3729-3733, 2007.

[24] X. Li, G. Ouyang, and D. A. Richards, "Predictability analysis of absence seizures with permutation entropy," Epilepsy Research, vol. 77, pp. 70-74, 2007.

[25] L. Zunino, D. G. Pérez, M. T. Martín, M. Garavaglia, A. Plastino, and O. A. Rosso, "Permutation entropy of fractional Brownian motion and fractional Gaussian noise," Phys. Lett. A, vol. 372, pp. 4768-4774, 2008.

[26] X. Li, S. Cui, and L. J. Voss, "Using permutation entropy to measure the electroencephalographic effects of sevoflurane," Anesthesiology, vol. 109, pp. 448-456, 2008.

[27] M. Matilla-García and M. Ruiz Marín, "A non-parametric independence test using permutation entropy," Journal of Econometrics, vol. 144, pp. 139-155, 2008

[28] O. A. Rosso and C. Masoller, "Detecting and quantifying stochastic and coherence resonances via information-theory complexity measurements," Phys. Rev. E, vol. 79, p. 040106(R), 2009.

[29] L. Zunino, M. Zanin, B. M. Tabak, D. G. Pérez, and O. A. Rosso, "Forbidden patterns, permutation entropy and stock market inefficiency," Physica A, vol. 388, pp. 2854-2864, 2009.

[30] L. D. Micco, H. A. Larrondo, A. Plastino, and O. A. Rosso, "Quantifiers for randomness of chaotic pseudo-random number generators," Phil. Trans. R. Soc. A, vol. 367, pp. 3281-3296, 2009.

[31] O. A. Rosso, H. A. Larrondo, M. T. Martín, A. Plastino, and M. A. Fuentes, "Distinguishing noise from chaos," Phys. Rev. Lett., vol. 99, p. 154102, 2007.

[32] J. M. Amigó, S. Zambrano, and M. A. F. Sanjuán, "True and false forbidden patterns in deterministic and random dynamics," Europhys. Lett., vol. 79, p. 50001, 2007.

[33] F. M. Atay, S. Jalan, and J. Jost, "Randomness, chaos, and structure," Complexity, vol. 15, pp. 29-35, 2009.

[34] X. Li and G. Ouyang, "Estimating coupling direction between neuronal populations with permutation conditional mutual information," Neuroimage, vol. 52, pp. 497-507, 2010.

[35] C. Bandt, "Ordinal time series analysis," Ecological modelling, vol. 182, pp. 229-238, 2005
[36] M. T. Rosenstein, J. J. Collins, and C. J. De Luca, "A practical method for calculating largest Lyapunov exponents from small data sets," Physica D, vol. 65, pp. 117-134, 1993.

[37] K. E. Chlouverakis, A. Argyris, A. Bogris, and D. Syvridis, "Complexity and synchronization in chaotic fiber-optic systems," Physica D, vol. 237, pp. $568-572,2008$.

[38] H. Kantz and T. Schreiber, Nonlinear time series analysis. Cambridge, UK: Cambridge University Press, 2004.

[39] J. D. Farmer, "Chaotic attractors of an infinite-dimensional dynamical system," Physica D, vol. 4, pp. 366-393, 1982.

[40] R. Hegger, M. J. Bünner, H. Kantz, and A. Giaquinta, "Identifying and modeling delay feedback systems," Phys. Rev. Lett., vol. 81, pp. 558$561,1998$.

[41] M. J. Bünner, M. Ciofini, A. Giaquinta, R. Hegger, H. Kantz, R. Meucci, and A. Politi, "Reconstruction of systems with delayed feedback: I. Theory," Eur. Phys. J. D, vol. 10, pp. 165-176, 2000.

[42] — " "Reconstruction of systems with delayed feedback: II. Application," Eur. Phys. J. D, vol. 10, pp. 177-187, 2000.

[43] K. Keller and M. Sinn, "A standardized approach to the KolmogorovSinai entropy," Nonlinearity, vol. 22, pp. 2417-2422, 2009.

[44] — "Kolmogorov-Sinai entropy from the ordinal viewpoint," Physica D, vol. 239, pp. 997-1000, 2010

[45] C. Bandt, G. Keller, and B. Pompe, "Entropy of interval maps via permutations," Nonlinearity, vol. 15, pp. 1595-1602, 2002.

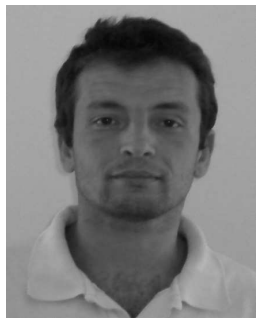

Luciano Zunino was born in Chivilcoy, Buenos Aires, Argentina, in 1975. He received the M.Sc. and $\mathrm{Ph} . \mathrm{D}$. degrees in Physics from the Universidad Nacional de La Plata, Buenos Aires, Argentina, in 2000 and 2005, respectively. Since April 2007, he has held a permanent research position at the Argentinean Consejo Nacional de Investigaciones Científicas y Técnicas (CONICET). His main research interests include time series analysis, nonlinear dynamics, econophysics and light propagation in turbulent media.

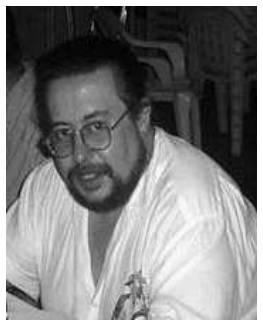

Osvaldo A. Rosso was born in Rojas, Buenos Aires, Argentina, on October 1954. He received the M.Sc and Ph.D. degrees in Physics from the Universidad Nacional de La Plata, Buenos Aires, Argentina, in 1978 an 1984, respectively. Since March 1985, he has held a permanent research position at the Argentinean Consejo Nacional de Investigaciones Científicas y Técnicas (CONICET). At present he is Professor Visitante do Exterior (PVE), categoria Doutor Senior, CAPES, Brazil (2010). He has authored or coauthored over 140 publications including about 96 journal papers. His main research interests include time series analysis, nonlinear dynamics, Information Theory, Time-Frequency Analysis and, their applications to physics, biological and medical sciences.

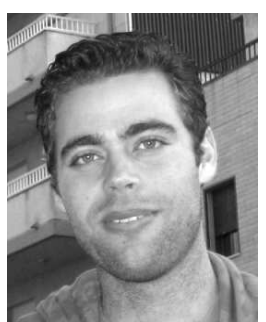

Miguel Cornelles Soriano was born in Benicarlo, Spain, in 1979. He received the Telecom Engineering degree from the Technical University of Catalonia, Spain, in 2002 and the Ph.D. degree in Engineering from the Free University of Brussels, Belgium, in 2006. Since January 2008, he holds a "Juan de la Cierva" scientific contract at the Instituto de Física Interdisciplinar y Sistemas Complejos (IFISC). His main research interests include optical feedback, theoretical modeling and synchronization properties of semiconductor lasers. 